\title{
Prevention of multiple pregnancies in couples with unexplained or mild male subfertility: randomised controlled trial of in vitro fertilisation with single embryo transfer or in vitro fertilisation in modified natural cycle compared with intrauterine insemination with controlled ovarian hyperstimulation
}

\author{
(c) $\underset{1}{(1)(8)}$ OPEN ACCESS
}

A J Bensdorp PhD student ${ }^{1}$, R I Tjon-Kon-Fat PhD student ${ }^{1}$, P M M Bossuyt clinical epidemiologist ${ }^{2}$, C A M Koks gynaecologist ${ }^{3}$, G J E Oosterhuis gynaecologist ${ }^{4}$, A Hoek gynaecologist ${ }^{5}$, P G A Hompes gynaecologist ${ }^{6}$, F J M Broekmans gynaecologist ${ }^{7}, \mathrm{H}$ R Verhoeve gynaecologist ${ }^{8}$, J P de Bruin gynaecologist ${ }^{9}, \mathrm{R}$ van Golde gynaecologist ${ }^{10}$, S Repping clinical embryologist ${ }^{1}$, B J Cohlen gynaecologist $^{11}$, M D A Lambers gynaecologist ${ }^{12}$, P F van Bommel gynaecologist ${ }^{13}$, E Slappendel clinical embryologist ${ }^{14}$, D Perquin gynaecologist ${ }^{15}$, J M Smeenk gynaecologist ${ }^{16}$, M J Pelinck gynaecologist $^{17}$, J Gianotten gynaecologist ${ }^{18}$, D A Hoozemans gynaecologist $^{19}$, J W M Maas gynaecologist $^{3}$, M J C Eijkemans statistician ${ }^{20}$, F van der Veen gynaecologist ${ }^{1}$, B W J Mol gynaecologist $^{21}$, M van Wely clinical epidemiologist ${ }^{1}$

\footnotetext{
${ }^{1}$ Centre for Reproductive Medicine, Academic Medical Centre, University of Amsterdam, 1100 DD Amsterdam, Netherlands; ${ }^{2}$ Department of Clinical Epidemiology, Biostatistics and Bioinformatics, Academic Medical Centre, University of Amsterdam; ${ }^{3}$ Máxima Medical Centre, Department of Obstetrics and Gynaecology, Veldhoven, Netherlands; ${ }^{4} \mathrm{St}$ Antonius Hospital, Department of Obstetrics and Gynaecology, Nieuwegein, Netherlands; ${ }^{5}$ University Medical Centre Groningen, University of Groningen, Department of Obstetrics and Gynaecology, Groningen, Netherlands; ${ }^{6} \mathrm{Vrije}$ Universiteit Medical Centre, Centre for Reproductive Medicine, Amsterdam; ' University Medical Centre Utrecht, Department for Reproductive Medicine, Utrecht, Netherlands; ${ }^{8}$ Onze Lieve Vrouwe Gasthuis, Department of Obstetrics and Gynaecology, Amsterdam, Netherlands; ' Jeroen Bosch Hospital, Department of Obstetrics and Gynaecology, 's Hertogenbosch, Netherlands; ${ }^{10}$ University Medical Centre Maastricht, Department of Obstetrics and Gynaecology, Maastricht, Netherlands; ${ }^{11}$ Isala Clinics, Department of Obstetrics and Gynaecology, Zwolle, Netherlands; ${ }^{12}$ Albert Schweitzer Hospital, Department of Obstetrics and Gynaecology, Dordrecht, Netherlands; ${ }^{13}$ Amphia Hospital, Department of Obstetrics and Gynaecology, Breda, Netherlands; ${ }^{14}$ Catharina Hospital, Department of Obstetrics and Gynaecology, Eindhoven, Netherlands; ${ }^{15}$ Medical Centre Leeuwarden, Obstetrics and Gynaecology, Leeuwarden, Netherlands; ${ }^{16}$ Elisabeth Hospital, Department of Obstetrics and Gynaecology, Tilburg, Netherlands; ${ }^{17}$ Scheper Hospital, Department of Obstetrics and Gynaecology, Emmen, Netherlands; ${ }^{18} \mathrm{Kennemer}$ Gasthuis, Department of Obstetrics and Gynaecology, Haarlem, Netherlands; ${ }^{19}$ Medical Spectrum Twente, Department of Obstetrics and Gynaecology, Enschede, Netherlands; ${ }^{20}$ University Medical Centre Utrecht, Julius Centre for Health Sciences and Primary Care, Utrecht, Netherlands; ${ }^{21}$ The Robinson Research Institute, School of Paediatrics and Reproductive Health, University of Adelaide, Adelaide, Australia
}

\section{Abstract}

Objectives To compare the effectiveness of in vitro fertilisation with single embryo transfer or in vitro fertilisation in a modified natural cycle with that of intrauterine insemination with controlled ovarian hyperstimulation in terms of a healthy child
Design Multicentre, open label, three arm, parallel group, randomised controlled non-inferiority trial.

Setting 17 centres in the Netherlands.

Participants Couples seeking fertility treatment after at least 12 months of unprotected intercourse, with the female partner aged between 18 
and 38 years, an unfavourable prognosis for natural conception, and a diagnosis of unexplained or mild male subfertility.

Interventions Three cycles of in vitro fertilisation with single embryo transfer (plus subsequent cryocycles), six cycles of in vitro fertilisation in a modified natural cycle, or six cycles of intrauterine insemination with ovarian hyperstimulation within 12 months after randomisation.

Main outcome measures The primary outcome was birth of a healthy child resulting from a singleton pregnancy conceived within 12 months after randomisation. Secondary outcomes were live birth, clinical pregnancy, ongoing pregnancy, multiple pregnancy, time to pregnancy, complications of pregnancy, and neonatal morbidity and mortality

Results 602 couples were randomly assigned between January 2009 and February 2012; 201 were allocated to in vitro fertilisation with single embryo transfer, 194 to in vitro fertilisation in a modified natural cycle, and 207 to intrauterine insemination with controlled ovarian

hyperstimulation. Birth of a healthy child occurred in 104 (52\%) couples in the in vitro fertilisation with single embryo transfer group, $83(43 \%)$ in the in vitro fertilisation in a modified natural cycle group, and 97 (47\%) in the intrauterine insemination with controlled ovarian hyperstimulation group. This corresponds to a risk, relative to intrauterine insemination with ovarian hyperstimulation, of 1.10 (95\% confidence interval 0.91 to 1.34) for in vitro fertilisation with single embryo transfer and $0.91(0.73$ to 1.14 ) for in vitro fertilisation in a modified natural cycle. These $95 \%$ confidence intervals do not extend below the predefined threshold of 0.69 for inferiority. Multiple pregnancy rates per ongoing pregnancy were $6 \%(7 / 121)$ after in vitro fertilisation with single embryo transfer, $5 \%$ $(5 / 102)$ after in vitro fertilisation in a modified natural cycle, and $7 \%$ (8/119) after intrauterine insemination with ovarian hyperstimulation (one sided $\mathrm{P}=0.52$ for in vitro fertilisation with single embryo transfer compared with intrauterine insemination with ovarian hyperstimulation; one sided $\mathrm{P}=0.33$ for in vitro fertilisation in a modified natural cycle compared with intrauterine insemination with controlled ovarian hyperstimulation).

Conclusions In vitro fertilisation with single embryo transfer and in vitro fertilisation in a modified natural cycle were non-inferior to intrauterine insemination with controlled ovarian hyperstimulation in terms of the birth of a healthy child and showed comparable, low multiple pregnancy rates.

Trial registration Current Controlled Trials ISRCTN52843371; Nederlands Trial Register NTR939.

\section{Introduction}

Involuntary childlessness affects more than 70 million couples worldwide and has a large impact on quality of life, leading to lasting psychosocial effects. ${ }^{12}$ Most couples will seek fertility care and consult their general practitioner for guidance.

Approximately half of these couples will be diagnosed as having unexplained or mild male subfertility. ${ }^{3}$ Most of them still have a good chance of conceiving and will achieve pregnancy without treatment. ${ }^{45}$ Treatment is thus indicated only if the chances of conceiving naturally are low and the success rate after treatment exceeds this probability. ${ }^{67}$

\section{Intrauterine insemination with controlled ovarian}

hyperstimulation is the first line fertility treatment in couples with an unfavourable prognosis for natural conception. ${ }^{89}$

However, concern exists about the increased rates of multiple pregnancy after intrauterine insemination with controlled ovarian hyperstimulation, as a result of the stimulation of multiple follicles. ${ }^{10}$ Intrauterine insemination with controlled ovarian hyperstimulation results in pregnancy rates of $13 \%$ per cycle at the expense of a multiple pregnancy rate that is estimated to be above $10 \%$ per ongoing pregnancy. ${ }^{611}$ Multiple pregnancies are associated with maternal morbidity such as pre-eclampsia, gestational diabetes, and a 50\% risk of premature delivery, resulting in considerable neonatal morbidity and mortality. ${ }^{12}$
In vitro fertilisation with single embryo transfer is increasingly implemented and might be equally effective but safer, as it prevents multiple pregnancies. ${ }^{13-15}$ Live birth rates with the transfer of one good quality embryo are $28 \%$ per cycle, with multiple pregnancy rates as low as $1 \%$ per live birth. ${ }^{16}$ The downside of in vitro fertilisation is that it is an invasive and burdensome procedure with higher costs. ${ }^{17}$

In vitro fertilisation in a modified natural cycle-a cycle in which monofollicular growth results in one oocyte at follicular aspiration and in one embryo after fertilisation - might be another treatment option. In vitro fertilisation with unstimulated or mildly stimulated regimens are becoming more popular. ${ }^{18-21}$ Cumulative live birth rates over six cycles of more than $30 \%$ have been reported, with almost no multiple pregnancies. ${ }^{22}$ In vitro fertilisation in a modified natural cycle has been advocated as less burdensome and less costly than "conventional" in vitro fertilisation. ${ }^{22} 23$

Multiple pregnancies seem to be prevented by in vitro fertilisation with single embryo transfer and in vitro fertilisation in a modified natural cycle, but this will be acceptable only if these interventions are as effective as intrauterine insemination with controlled ovarian hyperstimulation in terms of live birth rates. We therefore designed a randomised trial to evaluate the effectiveness of these interventions in terms of healthy children born from singleton pregnancies.

\section{Methods}

We did a multicentre, open label, three arm, parallel group, randomised controlled non-inferiority trial in 17 fertility clinics in the Netherlands between January 2009 and February 2013. Full details of the trial protocol can be found at www.studiesobsgyn.nl/ines.

\section{Participants}

Couples seeking fertility treatment after at least 12 months of unprotected intercourse were eligible. All participating couples provided written informed consent. All couples underwent basic fertility investigations, which included semen analysis, evaluation of ovulation, and tubal patency testing (Chlamydia antibody test, hysterosalpingography or laparoscopy). Inclusion criteria were age of female partner between 18 and 38 years, an unfavourable prognosis for natural conception, and a diagnosis of unexplained or mild male subfertility. We classified couples as having unexplained subfertility when the fertility investigations showed at least one patent fallopian tube, an ovulatory menstrual cycle, and a normal semen analysis (pre-wash total motile sperm count above 10 million) ${ }^{24}$ We considered couples who qualified for intrauterine insemination with donor sperm after at least six cycles of artificial intracervical insemination with donor sperm to have unexplained subfertility for the purpose of this study. Mild male subfertility was diagnosed when the semen analysis showed a pre-wash total motile sperm count between three and 10 million (according to the Dutch guidelines). We defined an unfavourable prognosis for natural conception as a probability of natural conception within the next 12 months of less than $30 \%$, as calculated with the validated synthesis model of Hunault. ${ }^{7}$ This model encompasses female age, duration of subfertility, whether subfertility is primary or secondary, percentage of motile progressive sperm, and referral status. It is readily available for all clinicians (www.freya.nl/web_bereken/bereken.php). Exclusion criteria were anovulation, double sided tubal disease, severe endometriosis, premature ovarian failure, and known 
endocrine disorders (such as Cushing's syndrome or adrenal hyperplasia).

\section{Randomisation and masking}

Couples were randomly allocated in a 1:1:1 ratio to receive either three consecutive cycles of in vitro fertilisation with single embryo transfer plus subsequent cryocycles, six consecutive cycles of in vitro fertilisation in a modified natural cycle, or six consecutive cycles of intrauterine insemination with controlled ovarian hyperstimulation within 12 months. Randomisation was performed with an online randomisation program, using biased coin minimisation, stratified for study centre. A web based program generated a unique number with allocation code after entry of the patient's initials and date of birth. Neither the recruiters nor the trial project group could access the randomisation sequence. Blinding was not possible owing to the nature of the interventions.

\section{Interventions}

In the in vitro fertilisation with single embryo transfer group, participating hospitals could adhere to local stimulation protocols, which were either long/short agonist or antagonist protocols. Controlled ovarian hyperstimulation started with 150 IU follicle stimulating hormone. Treatment was continued until at least two follicles of at least $18 \mathrm{~mm}$ had developed. Ovulation triggering was induced by 10000 IU human chorionic gonadotropin hormone (Pregnyl, Merck Sharp \& Dohme), and cumulus-oocyte complexes were recovered by transvaginal ultrasound guided retrieval 36 hours thereafter.

We adhered to an elective single embryo transfer policy: if one good quality embryo was available, we transferred one embryo. ${ }^{25}$ If more than one good quality embryo was available, suitable surplus embryos were cryopreserved. If no good quality embryos were available, two embryos would be transferred. For the morphological score, the degree of fragmentation of the embryo and the uniformity of the blastomeres were assessed daily. ${ }^{26}$ The embryo was given a score of 1 (no fragmentation), $2(<20 \%$ fragmentation), 3 (20-50\% fragmentation), or 4 (>50\% fragmentation). We defined good quality embryos as those with a cumulative embryo score of 24 or higher. Embryo transfer followed on day three. All available frozen embryos were transferred after thawing before a new treatment cycle was started.

During our trial, the results of a pilot study, randomising women to three cycles of intrauterine insemination with controlled ovarian hyperstimulation or one cycle of in vitro fertilisation with single embryo transfer, was published. This pilot study showed that the policy of transferring two embryos when no good quality embryos are available is not effective in preventing multiple pregnancies. ${ }^{27} \mathrm{We}$ amended our study protocol, and from February 2010, after allocation of 48 women to the in vitro fertilisation with single embryo transfer group, a strict single embryo transfer policy was implemented (that is, single embryo transfer was performed irrespective of the quality of the embryo).

In the in vitro fertilisation in a modified natural cycle group, women were monitored by transvaginal ultrasound from days eight to 10 of the cycle onward; when the lead follicle had a mean diameter of at least $14 \mathrm{~mm}$, they were given daily injections of $0.25 \mathrm{mg}$ of the gonadotropin releasing hormone antagonist to prevent premature ovulation together with $150 \mathrm{IU}$ follicle stimulating hormone to prevent collapse of the follicle and a concomitant fall in oestradiol concentrations. Follicle stimulating hormone was continued up to the day of the ovulation triggering, and the gonadotropin releasing hormone antagonist was last given on the day of ovulation triggering. When a follicle with a diameter of 17-18 mm was observed, ovulation triggering was achieved by subcutaneous injection of $10000 \mathrm{IU}$ of human chorionic gonadotropin. Oocyte retrieval was planned 34 hours thereafter and was performed without anaesthesia or sedation. If an oocyte was obtained and fertilised, the embryo was transferred on day three. For luteal support, human chorionic gonadotropin 1500 IU was given by subcutaneous injections on days five, eight, and 11 after oocyte retrieval. The next treatment cycle could start immediately after the previous cycle.

In the intrauterine insemination with controlled ovarian hyperstimulation group, women received controlled ovarian hyperstimulation according to the local protocol, with either $100 \mathrm{mg}$ clomiphene citrate (cycle day three to seven) or daily subcutaneous injections of 75 IU follicle stimulating hormone (starting dose). Follicular growth was monitored by ultrasound; when at least one follicle of 17 or $18 \mathrm{~mm}$ was present, final oocyte maturation was induced by the administration of 5000 IU human chorionic gonadotropin. Approximately 36 hours thereafter, intrauterine insemination was performed. Intrauterine insemination with controlled ovarian hyperstimulation cycles were cancelled when there were more than three follicles with a diameter of $16 \mathrm{~mm}$ or more than five follicles with a diameter of $12 \mathrm{~mm}$. Those couples were instructed to refrain from unprotected intercourse. The box summarises the three interventions.

\section{Follow-up}

Couples were followed up for 12 months after randomisation. We included all interventions that couples received within 12 months after randomisation. A pregnancy test was performed two weeks after embryo transfer or intrauterine insemination. Clinical and ongoing pregnancies were confirmed by ultrasonography. If a miscarriage occurred within 12 months after randomisation, couples could continue their allocated treatment. Natural conceptions were also included in the analysis in the three groups.

\section{Outcomes}

The primary outcome was the birth of a healthy child, resulting from a singleton pregnancy conceived within 12 months after randomisation. A child was considered healthy when born at term, defined as a gestational age between 37 and 42 weeks; with a birth weight above the fifth centile according to the Dutch reference curves corrected for parity, sex, and ethnicity; without congenital anomalies; and developing normally up to six weeks after birth. ${ }^{28}$ This information was collected from the parents, child health centres, or paediatricians.

Secondary outcomes were any live birth, multiple pregnancy (registered heartbeat of at least two fetuses at 12 weeks of gestation), clinical pregnancy (any registered embryonic heartbeat on ultrasound), ongoing pregnancy (registered heartbeat of a fetus at 12 weeks of gestation), neonatal mortality (death of the child within 28 days after birth), neonatal morbidity (preterm birth $<37$ weeks, birth weight $<2500 \mathrm{~g}$ ), pregnancy complications, (pregnancy induced hypertension, (pre-)eclampsia, HELLP syndrome), and time to pregnancy. All serious adverse events were reported to the trial coordinator. An independent data safety and monitoring committee (consisting of two gynaecologists and one statistician) monitored the progress of the study and the safety of the women and did 


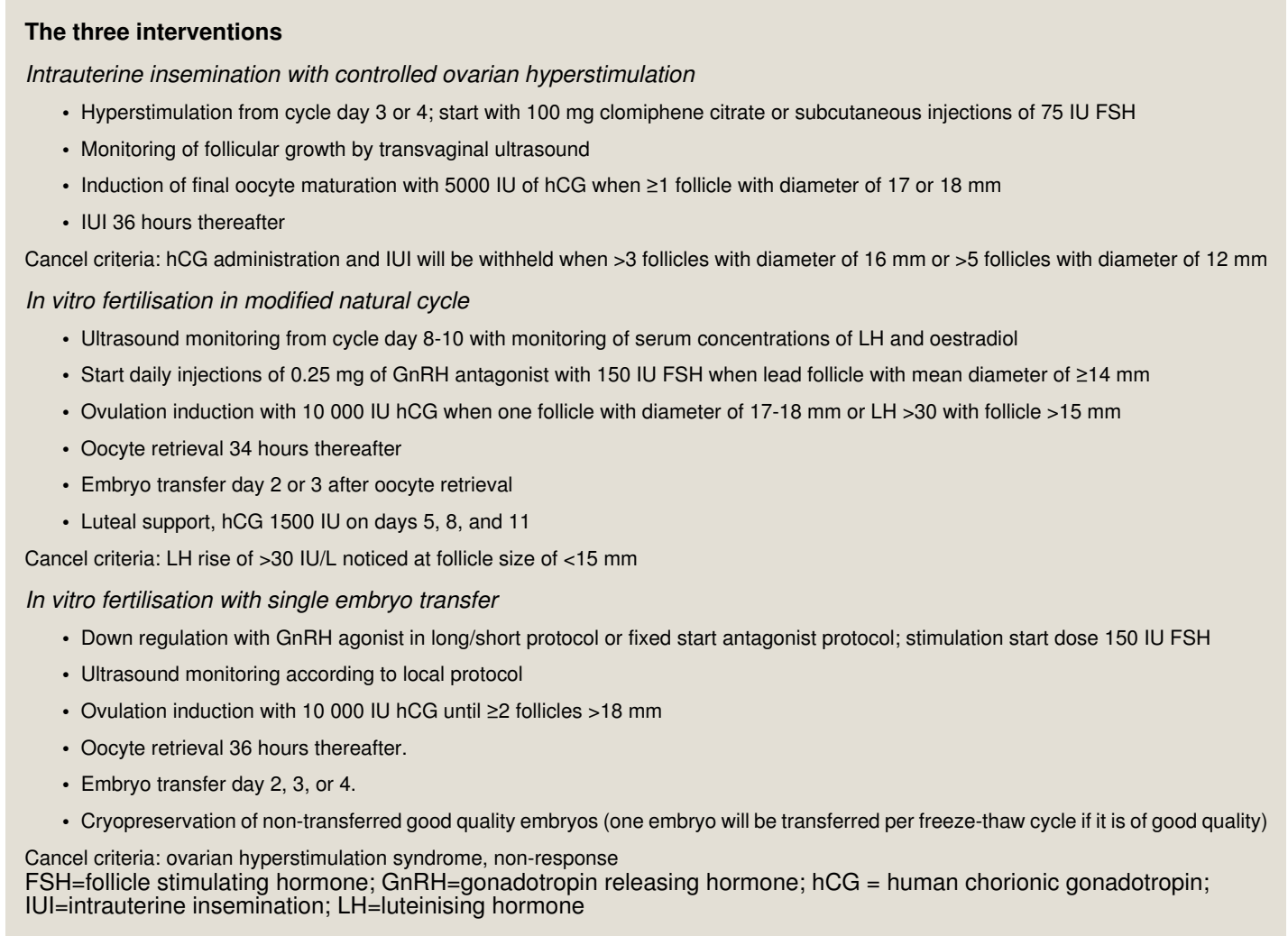

a blinded interim analysis to exclude large differences after 300 inclusions with ongoing pregnancy as a surrogate outcome.

\section{Study design, sample size, and statistical analysis}

The trial was designed as a non-inferiority trial. The null hypothesis assumed that the pregnancy rates would be comparable between all interventions. We expected $40 \%$ of the couples to have a live birth within 12 months after starting with intrauterine insemination with controlled ovarian hyperstimulation. ${ }^{29}$ For the power calculation, using a 5\% significance level, we needed 190 couples to achieve $80 \%$ power to exclude a difference of $12.5 \%$ or more to the detriment of in vitro fertilisation with single embryo transfer and in vitro fertilisation in a modified natural cycle. This difference corresponds to a relative risk of 0.69 . To account for a $5 \%$ loss to follow-up, we needed to include 200 couples in each group. All randomised patients were included in all analyses. We estimated differences in the primary and secondary outcomes as relative risks with $95 \%$ confidence intervals. We constructed Kaplan-Meier survival curves in each treatment group for the time to ongoing pregnancy with a healthy child. We used SPSS (version 20.0) for all statistical analyses. We considered $P$ values below 0.05 as indicating statistically significant differences.

\section{Results}

Between January 2009 and February 2012, 869 couples fulfilled the inclusion criteria and were invited to the trial, of whom 267 declined randomisation. We included 602 couples: 201 couples were assigned to in vitro fertilisation with single embryo transfer, 194 to in vitro fertilisation in a modified natural cycle, and 207 to intrauterine insemination with controlled ovarian hyperstimulation (fig $1 \Downarrow$ ). Baseline characteristics in the three groups were comparable (table $1 \Downarrow$ ).
Tables $2 \Downarrow$ and $3 \Downarrow$ list all interventions that took place during the 12 months' follow-up. Table $2 \Downarrow$ shows the number of couples who received the allocated treatment per cycle, and table $3 \Downarrow$ shows the total number of cycles within 12 months. Table $2 \Downarrow$ also shows additional treatments that couples received when they switched from the allocated treatment to another treatment or when they continued with another treatment after having completed their allocated treatment within 12 months after randomisation.

In the in vitro fertilisation with single embryo transfer group, $15 \%(30 / 201)$ of the couples discontinued treatment before receiving three fresh cycles of in vitro fertilisation with single embryo transfer or achieving pregnancy. Discontinuation rates were $23 \%(45 / 194)$ in the in vitro fertilisation in a modified natural cycle group and $13 \%$ (26/207) in the intrauterine insemination with controlled ovarian hyperstimulation group. These couples discontinued treatment because they switched to another treatment or chose to end treatment.

\section{Pregnancy outcomes}

Table $4 \Downarrow$ lists the outcomes of pregnancy. In all, 104 (52\%) couples had healthy children after in vitro fertilisation with single embryo transfer, $83(43 \%)$ after in vitro fertilisation in a modified natural cycle, and 97 (47\%) after intrauterine insemination with controlled ovarian hyperstimulation. This corresponds to a relative risk, compared with intrauterine insemination with controlled ovarian hyperstimulation, of 1.10 (95\% confidence interval 0.91 to 1.34 ) for in vitro fertilisation with single embryo transfer and 0.91 (0.73 to 1.14) for in vitro fertilisation in a modified natural cycle. These $95 \%$ confidence intervals do not extend below the predefined threshold of 0.69 for inferiority.

We found no evidence of a difference between the groups in rates of live birth, ongoing pregnancy, or clinical pregnancy. Multiple pregnancy rates were $6 \%$ after in vitro fertilisation with single embryo transfer, $5 \%$ after in vitro fertilisation in a 
modified natural cycle, and 7\% after intrauterine insemination with controlled ovarian hyperstimulation (one sided $\mathrm{P}=0.52$ for in vitro fertilisation with single embryo transfer compared with intrauterine insemination with controlled ovarian hyperstimulation; one sided $\mathrm{P}=0.33$ for in vitro fertilisation in a modified natural cycle compared with intrauterine insemination with controlled ovarian hyperstimulation). One triplet pregnancy occurred in the intrauterine insemination with controlled ovarian hyperstimulation group; all other multiple pregnancies were twin pregnancies.

In the 48 couples who received in vitro fertilisation with single embryo transfer before the amendment of the protocol, 22 (46\%) women had a healthy singleton child and three $(6 \%)$ women had healthy twins. In the 153 couples who received strict in vitro fertilisation with single embryo transfer after the amendment of the protocol, 82 (54\%) women had a healthy singleton and four (3\%) women had twins.

\section{Neonatal outcomes}

Neonatal death did not occur in this study. We found no differences between groups in the relative number of preterm babies or in those with low birth weight $(<2500 \mathrm{~g})$ (table $4 \Downarrow)$. Congenital anomalies were reported in 10 cases (table $4 \Downarrow$ ). According to the ICD-10 (international classification of diseases, 10th revision) classification, one case of an aortic stenosis (Q23) occurred in the in vitro fertilisation with single embryo transfer group. One case of an urachus fistula (Q64), one case of Moebius syndrome (Q87), and two cases of Down's syndrome (Q90) occurred in the in vitro fertilisation in a modified natural cycle group. In the intrauterine insemination with controlled ovarian hyperstimulation group, one case of patent/persistent foramen ovale (Q21), one case of congenital deafness (H90), one case of an umbilical hernia (K42), one case of Down's syndrome (Q90), and one case of oesophageal atresia (Q39) occurred.

\section{Maternal outcomes}

Two women in the in vitro fertilisation with single embryo transfer group and one woman in the intrauterine insemination with controlled ovarian hyperstimulation group developed ovarian hyperstimulation syndrome, and all were managed expectantly: two were managed in an outpatient setting, and one was admitted for one night. Pregnancy induced hypertension, pre-eclampsia, and HELLP syndrome occurred at similar frequencies in the three groups (table $4 \Downarrow$ ).

\section{Time to pregnancy}

Average time to pregnancy leading to a healthy child was 8.04 months for in vitro fertilisation with single embryo transfer, 8.32 months for in vitro fertilisation in a modified natural cycle, and 8.39 months for intrauterine insemination with controlled ovarian hyperstimulation (fig $2 \Downarrow$ ). The differences were not statistically significant for in vitro fertilisation with single embryo transfer (log rank: $\mathrm{P}=0.38$ ) or for in vitro fertilisation in a modified natural cycle (log rank: $\mathrm{P}=0.59)$ compared with intrauterine insemination with controlled ovarian hyperstimulation.

Figure $3 \Downarrow$ shows time to ongoing pregnancy within 12 months after randomisation, classified as to whether the pregnancy was achieved with the allocated treatment, with additional treatment, or after natural conception. Twenty four (12\%) couples had a live birth after natural conception in the in vitro fertilisation with single embryo transfer group, $30(15 \%)$ in the in vitro fertilisation in a modified natural cycle group, and $22(11 \%)$ in the intrauterine insemination with controlled ovarian hyperstimulation group. Of the pregnancies achieved through allocated treatment in the in vitro fertilisation with single embryo transfer group, 68 (76\%) were ongoing pregnancies after fresh embryo transfer and 21 (24\%) after frozen embryo transfer.

\section{Discussion}

In this multicentre, open label, three arm, parallel group, randomised controlled non-inferiority trial, we showed that in vitro fertilisation with single embryo transfer and in vitro fertilisation in a modified natural cycle are non-inferior to intrauterine insemination with controlled ovarian hyperstimulation in terms of the birth of a healthy child in couples with unexplained or mild male subfertility and unfavourable fertility prospects. Rates of multiple pregnancy in all three treatment arms were comparable and amounted to less than $7 \%$. Also, time to pregnancy was comparable in all arms.

\section{Strengths and weaknesses of study}

Couples were treated with three cycles of in vitro fertilisation with single embryo transfer, six cycles of in vitro fertilisation in a modified natural cycle, or six cycles of intrauterine insemination with controlled ovarian hyperstimulation during 12 months to determine the chances of pregnancy over a realistic time period following treatment, reflecting daily clinical practice. Also, all additional interventions or pregnancies achieved through natural conception within the 12 months after randomisation were registered and included in the analysis.

The trial was further strengthened by our primary outcome, the birth of a single healthy child. The European Society for Human Reproduction and Embryology (ESHRE) has recommended this outcome measure, as the primary aim of reproductive medicine is to help couples with an unfulfilled wish for a child to have a healthy child.$^{30}$ Our criteria for a healthy child were quite strict, but if these were softened to include healthy late preterm infants, more children born out of a twin pregnancy in our study would be considered healthy.

We initially chose elective single embryo transfer on the basis of data from a meta-analysis that showed that elective single embryo transfer in patients with a good prognosis resulted in a higher chance of delivering a term singleton live birth, compared with double embryo transfer. Birth rates overall were lower, but this could almost be compensated by additional cycles of frozen single embryo transfer..$^{31}$ Our change in policy from elective single embryo transfer to a policy of strict single embryo transfer did not lead to lower pregnancy rates but did lead to lower multiple pregnancy rates. The four dizygotic twin pregnancies after the policy change occurred after double embryo transfer at the request of the patients. This shows the difficulty in maintaining a rigid policy of in vitro fertilisation with single embryo transfer and in vitro fertilisation in a modified natural cycle.

The margin of equivalence, $12.5 \%$, may be seen as relatively high, as one could argue that a $5 \%$ or $10 \%$ increase may be important in clinical practice. We hypothesised that the pregnancy rates of in vitro fertilisation had to be considerably better than those of intrauterine insemination with controlled ovarian hyperstimulation, as in vitro fertilisation is a more invasive and expensive treatment, and we considered $12.5 \%$ to be reasonable in this respect. 


\section{Generalisability}

We included only couples with chances of conceiving below $30 \%$, as it has been established that intrauterine insemination with controlled ovarian hyperstimulation is not effective in couples with chances of natural conception greater than $30 \%$ according to the Hunault model. ${ }^{6}$ The use of this prediction model obviates the need to minimise by female age, parity, and duration of subfertility, as all these factors are incorporated in the model.

We included only couples with women aged under 38 years, as this age limit had often been used in studies comparing single embryo transfer with double embryo transfer in couples with good prognosis. ${ }^{32-35}$ As a consequence, we did not want to expose couples in which the woman is at the end of her reproductive period to single embryo transfer.

\section{Interpretation in context of setting and intervention}

Results from this trial partly confirm the findings of a meta-analysis. ${ }^{36}$ This Cochrane review included four trials, and the results suggested that among treatment naïve couples with unexplained subfertility, no significant difference existed in live birth rates for intrauterine insemination with controlled ovarian hyperstimulation versus in vitro fertilisation. However, two recent trials concluded that pregnancy was achieved faster with immediate in vitro fertilisation. ${ }^{37}{ }^{38}$ Apart from differences in population, the stimulation protocols were more aggressive than ours, and the multiple pregnancy rates were higher. We found no significant difference in time to pregnancy in the three arms of our study, so no time is wasted by starting with intrauterine insemination with controlled ovarian hyperstimulation.

Many couples stop treatment or switch to another treatment before an ongoing pregnancy is achieved. By allowing couples to receive other treatments if they did not achieve an ongoing pregnancy leading to a live birth after in vitro fertilisation with single embryo transfer, in vitro fertilisation in a modified natural cycle, or intrauterine insemination with controlled ovarian hyperstimulation within a time horizon of one year, our study design reflects daily practice and leads to high external validity.

As we have excluded large differences in efficacy and safety between in vitro fertilisation with single embryo transfer and in vitro fertilisation in a modified natural cycle compared with intrauterine insemination with controlled ovarian hyperstimulation, costs and patients' preferences should be considered. The number of couples who discontinue treatment if a pregnancy does not occur can be considered a proxy for the burden of treatment. In this trial, in vitro fertilisation in a modified natural cycle had a higher rate of couples discontinuing treatment. From that perspective, in vitro fertilisation in a modified natural cycle might not be as patient friendly as previously considered. ${ }^{22}$

The number of multiple pregnancies in the intrauterine insemination with controlled ovarian hyperstimulation group was lower in this trial than previously reported. ${ }^{39-41}$ We aimed to achieve a maximum of three dominant follicles and used strict cancellation criteria. This resulted in low chances of multiple pregnancy while maintaining live birth rates, indicating the relative safety of intrauterine insemination with controlled ovarian hyperstimulation when strict criteria are maintained. ${ }^{11}$ Our rather cautious approach to stimulation also resulted in induction of ovulation and subsequent intrauterine insemination when only one mature follicle was present. Furthermore, intrauterine insemination with controlled ovarian hyperstimulation was performed with either clomiphene citrate or follicle stimulation hormone; this may have influenced the rates of both pregnancy and multiple pregnancy. Whether the use of clomiphene citrate should be preferred over follicle stimulating hormone in intrauterine insemination with controlled ovarian hyperstimulation cycles in couples with unexplained subfertility is being studied in a randomised controlled (Dutch trial registration (www.trialregister.nl) number 4057).

We found comparable rates of multiple pregnancy between the three interventions. This would suggest that in vitro fertilisation with single embryo transfer or in vitro fertilisation in a modified natural cycle has no benefit in the reduction of multiple pregnancies over intrauterine insemination with controlled ovarian hyperstimulation. However, if a twin pregnancy must be avoided at all costs, intrauterine insemination with controlled ovarian hyperstimulation should not be performed, as a small risk of a multiple pregnancy still exists. In vitro fertilisation with the transfer of a single embryo, irrespective of embryo quality, should be the treatment of choice, whether after in vitro fertilisation in a modified natural cycle or in vitro fertilisation in a modified natural cycle with conventional ovarian hyperstimulation. To the detriment of in vitro fertilisation in a modified natural cycle, no surplus embryos are available for cryopreservation.

Recently, the question was posed as to whether couples with unexplained subfertility and an unfavourable prognosis for natural conception should be treated at all. The updated 2013 guideline from the National Institute for Health and Care Excellence abandoned intrauterine insemination with controlled ovarian hyperstimulation for these couples and recommended extended expectant management for all couples with unexplained subfertility instead, on the basis of a lack of data indicating efficacy of intrauterine insemination for unexplained subfertility. ${ }^{42}$ However, trials comparing intrauterine insemination with controlled ovarian hyperstimulation versus extended expectant management in couples with an unfavourable prognosis on natural conception have not yet been performed. We believe that such a trial is necessary before such guidelines are implemented.

\section{Conclusions}

We have shown that in vitro fertilisation with single embryo transfer and in vitro fertilisation in a modified natural cycle were non-inferior to intrauterine insemination with controlled ovarian hyperstimulation in terms of the birth of a healthy child, with comparable multiple pregnancy rates. The absence of a marked difference in pregnancy outcomes suggests that the more invasive in vitro fertilisation with single embryo transfer and in vitro fertilisation in a modified natural cycle may not be desirable alternatives to intrauterine insemination with controlled ovarian hyperstimulation. In view of these results, there seems no reason to abandon intrauterine insemination with controlled ovarian hyperstimulation as a first line treatment of couples with unexplained or mild male subfertility and an unfavourable prognosis for natural conception.

We thank all the couples who participated in this study. We also thank all the members of the Dutch Consortium (www.studies-obsgyn.nl), especially the research nurses and midwives, for all their hard work and dedication. We thank $S$ Mastenbroek for all his support with the construction of the database and M Kruijt, Z van Dijk, and C Crul for their support during this trial.

BWJM now works at the Robinson Institute, School of Paediatrics and Reproductive Health, University of Adelaide, Australia. 


\section{What is already known on this topic}

Up to half of subfertile couples are diagnosed as having unexplained or mild male subfertility

If these couples have unfavourable chances of natural conception, intrauterine insemination with controlled ovarian hyperstimulation is the first line treatment

In vitro fertilisation with single embryo transfer or in vitro fertilisation in a modified natural cycle are alternative treatments that may prevent multiple pregnancies

\section{What this study adds}

In vitro fertilisation with single embryo transfer and in vitro fertilisation in a modified natural cycle were non-inferior to intrauterine insemination with controlled ovarian hyperstimulation in terms of the birth of a healthy child

Rates of multiple pregnancy were comparable and low in the three group

There seems to be no reason to abandon intrauterine insemination with controlled ovarian hyperstimulation as a first line treatment of couples with unexplained or mild male subfertility and an unfavourable prognosis for natural conception

Contributors: All authors acquired the data from the participating centres, provided critical discussion, and contributed in the preparation of the manuscript. MvW and FvdV designed the trial, and AJB was the trial coordinator. AJB and RIT participated in the analysis and in the drafting of the manuscript. MJCE supervised the analyses. MvW, FvdV, and BWJM participated in the analysis, manuscript drafting, and supervision of the work. MvW is the guarantor.

Funding: The study was supported by a grant from ZonMW, the Dutch Organization for Health Research and Development (120620027) and a grant from Zorgverzekeraars Nederland, the Dutch association of healthcare insurers (09-003). The funders had no role in study design or in collection, analysis, or interpretation of the data.

Competing interests: All authors have completed the ICJME unified disclosure form at www.icmje.org/coi_disclosure.pdf (available on request from the corresponding author) and declare: the work was funded partly by ZonMW the Dutch Organization for Health Research and Development and a grant from Zorgverzekeraars Nederland, the Dutch association of healthcare insurers; no financial relationships with any organisations that might have an interest in the submitted work in the previous three years; no other relationships or activities that could appear to have influenced the submitted work.

Ethical approval: The study protocol was approved by the Medical Ethical Committee of the Academic Medical Centre in Amsterdam (MEC 07-064) and the boards of directors of each participating centre. All participating couples provided written informed consent.

Transparency: MvW affirms that the manuscript is an honest, accurate, and transparent account of the study being reported; that no important aspects of the study have been omitted; and that any discrepancies from the study as planned (and, if relevant, registered) have been explained.

Data sharing: The INeS trial project group supports the policy of making relevant anonymised patient level data available on reasonable request. Requests should be directed to m.vanwely@amc.nl.

1 Boivin J, Bunting L, Collins JA, Nygren KG. International estimates of infertility prevalence and treatment-seeking: potential need and demand for infertility medical care. Hum Reprod 2007;22:1506-12.

2 Wirtberg I, Möller A, Hogström L, Tronstad S-E, Lalos A. Life 20 years after unsuccessful infertility treatment. Hum Reprod 2007;22:598-604.

3 Brandes M, Hamilton CJ, de Bruin JP, Nelen WL, Kremer JA. The relative contribution of IVF to the total ongoing pregnancy rate in a subfertile cohort. Hum Reprod 2010;25:118-26.

4 Brandes M, Hamilton CJ, van der Steen JO, de Bruin JP, Bots RS, Nelen WL, et al. Unexplained infertility: overall ongoing pregnancy rate and mode of conception. Hum Reprod 2011;26:360-8.

5 Evers JL. Female subfertility. Lancet 2002;360:151-9

6 Steures P, van der Steeg JW, Hompes PG, Habbema JD, Eijkemans MJ, Broekmans FJ, et al. Intrauterine insemination with controlled ovarian hyperstimulation versus expectan management for couples with unexplained subfertility and an intermediate prognosis: a randomised clinical trial. Lancet 2006:368:216-21.

7 Van der Steeg JW, Steures P, Eijkemans MJ, Habbema JD, Hompes PG, Broekmans $\mathrm{FJ}$, et al. Pregnancy is predictable: a large-scale prospective external validation of the prediction of spontaneous pregnancy in subfertile couples. Hum Reprod 2007;22:536-42.

8 Bensdorp AJ, Cohlen BJ, Heineman MJ, Vandekerckhove P. Intra-uterine insemination for male subfertility. Cochrane Database Syst Rev 2007;4:CD000360.

9 Veltman-Verhulst SM, Cohlen BJ, Hughes E, Heineman MJ. Intra-uterine insemination for unexplained subfertility. Cochrane Database Syst Rev 2012;9:CD001838.

10 Fauser BC, Devroey P, Macklon NS. Multiple birth resulting from ovarian stimulation for subfertility treatment. Lancet 2005;365:1807-16.
11 Van Rumste MM, Custers IM, van der Veen F, van Wely M, Evers JL, Mol BW. The influence of the number of follicles on pregnancy rates in intrauterine insemination with ovarian stimulation: a meta-analysis. Hum Reprod Update 2008;14:563-70.

12 Ombelet W, De Sutter P, Van der Elst J, Martens G. Multiple gestation and infertility treatment: registration, reflection and reaction-the Belgian project. Hum Reprod Update 2005:11:3-14

13 Mansour R, Ishihara O, Adamson GD, Dyer S, de Mouzon J, Nygren KG, et al. International Committee for Monitoring Assisted Reproductive Technologies world report: assisted reproductive technology 2006. Hum Reprod 2014;29:1536-51.

14 Sullivan EA, Zegers-Hochschild F, Mansour R, Ishihara O, de Mouzon J, Nygren KG, et al. International Committee for Monitoring Assisted Reproductive Technologies (ICMART) world report: assisted reproductive technology 2004. Hum Reprod 2013:28:1375-90.

15 Practice Committee of the American Society for Reproductive Medicine. Multiple gestation associated with infertility therapy: an American Society for Reproductive Medicine Practice Committee opinion. Fertil Steril 2012;97:825-34

16 Pandian Z, Marjoribanks J, Ozturk O, Serour G, Bhattacharya S. Number of embryos for transfer following in vitro fertilisation or intra-cytoplasmic sperm injection. Cochrane Database Syst Rev 2013;7:CD003416.

17 Boivin J, Griffiths E, Venetis CA. Emotional distress in infertile women and failure of assisted reproductive technologies meta-analysis of prospective psychosocial studies. BMJ 2011;342:1-9.

18 Aanesen A, Nygren K-G, Nylund L. Modified natural cycle IVF and mild IVF: a 10 year Swedish experience. Reprod Biomed Online 2010;20:156-62.

19 Gordon JD, Dimattina M, Reh A, Botes A, Celia G, Payson M. Utilization and success rates of unstimulated in vitro fertilization in the United States: an analysis of the Society for Assisted Reproductive Technology database. Fertil Steril 2013;100:392-5.

20 Matsuura T, Takehara Y, Kaijima H, Teramoto S, Kato O. Natural IVF cycles may be desirable for women with repeated failures by stimulated IVF cycles. J Assist Reprod Genet 2008;25:163-7.

21 Schimberni M, Morgia F, Colabianchi J, Giallonardo A, Piscitelli C, Giannini P, et al. Natural-cycle in vitro fertilization in poor responder patients: a survey of 500 consecutive cycles. Fertil Steril 2009;92:1297-301.

22 Pelinck MJ, Vogel NE, Arts EG, Simons AH, Heineman MJ, Hoek A. Cumulative pregnancy rates after a maximum of nine cycles of modified natural cycle IVF and analysis of patien drop-out: a cohort study. Hum Reprod 2007;22:2463-70.

23 Verberg MF, Macklon NS, Nargund G, Frydman R, Devroey P, Broekmans FJ, et al. Mild ovarian stimulation for IVF. Hum Reprod Update 2009;15:13-29.

24 Bensdorp AJ, Slappendel E, Koks C, Oosterhuis J, Hoek A, Hompes P, et al. The INeS study: prevention of multiple pregnancies: a randomised controlled trial comparing IUI $\mathrm{COH}$ versus IVF e SET versus MNC IVF in couples with unexplained or mild male subfertility. BMC Womens Health 2009;9:35.

25 Practice Committee of the Society for Assisted Reproductive Technology, Practice Medicine Committee of the American Society for Reproductive Medicine. Elective single-embryo transfer. Fertil Steril 2012:97:835-42.

26 Puissant F, Van Rysselberge M, Barlow P, Deweze J, Leroy F. Embryo scoring as a prognostic tool in IVF treatment. Hum Reprod 1987;2:705-8.

27 Custers IM, König TE, Broekmans FJ, Hompes PG, Kaaijk E, Oosterhuis J, et al. Couples with unexplained subfertility and unfavorable prognosis: a randomized pilot trial comparing the effectiveness of in vitro fertilization with elective single embryo transfer versus intrauterine insemination with controlled ovarian stimulation. Fertil Steril 2011;96:1107-11.e1.

28 Visser GH, Eilers PH, Elferink-Stinkens PM, Merkus HM, Wit JM. New Dutch reference curves for birthweight by gestational age. Early Hum Dev 2009;85:737-44.

29 Steures P, van der Steeg JW, Hompes PG, van der Veen F, Mol BW. Intrauterine insemination in the Netherlands. Reprod Biomed Online 2007;14:110-6.

30 Land JA. Risks and complications in assisted reproduction techniques: report of an ESHRE consensus meeting. Hum Reprod 2003;18:455-7.

31 Pandian Z, Templeton A, Serour G, Bhattacharya S. Number of embryos for transfer after IVF and ICSI: a Cochrane review. Hum Reprod 2005;20:2681-7.

32 Lukassen HG, Braat DD, Wetzels AM, Zielhuis GA, Adang EM, Scheenjes E, et al. Two cycles with single embryo transfer versus one cycle with double embryo transfer: a randomized controlled trial. Hum Reprod 2005;20:702-8.

33 Gerris J, De Sutter P, De Neubourg D, Van Royen E, Vander Elst J, Mangelschots K, et al. A real-life prospective health economic study of elective single embryo transfer versus two-embryo transfer in first IVF/ICSI cycles. Hum Reprod 2004;19:917-23.

34 Van Montfoort AP, Dumoulin JC, Land JA, Coonen E, Derhaag JG, Evers JL. Elective single embryo transfer (eSET) policy in the first three IVF/ICSI treatment cycles. Hum Reprod 2005;20:433-6.

35 Thurin A, Hausken J, Hillensjö T, Jablonowska B, Pinborg A, Strandell A, et al. Elective single-embryo transfer versus double-embryo transfer in in vitro fertilization. N Engl J Med 2004;351:2392-402

36 Pandian Z, Gibreel A, Bhattacharya S. In vitro fertilisation for unexplained subfertility. Cochrane Database Syst Rev 2012;4:CD003357. 
37 Reindollar RH, Regan MM, Neumann PJ, Levine B-S, Thornton KL, Alper MM, et al. A randomized clinical trial to evaluate optimal treatment for unexplained infertility: the fast track and standard treatment (FASTT) trial. Fertil Steril 2010;94:888-99.

38 Goldman MB, Thornton KL, Ryley D, Alper MM, Fung JL, Hornstein MD, et al. A randomized clinical trial to determine optimal infertility treatment in older couples: the Forty and Over Treatment Trial (FORT-T). Fertil Steril 2014;101:1574-81.e1-2.

39 Goverde AJ, McDonnell J, Vermeiden JP, Schats R, Rutten FF, Schoemaker J. Intrauterine insemination or in-vitro fertilisation in idiopathic subfertility and male subfertility: a randomised trial and cost-effectiveness analysis. Lancet 2000;355:13-8.

40 Gleicher N, Oleske DM, Tur-Kaspa I, Vidali A, Karande V. Reducing the risk of high-order multiple pregnancy after ovarian stimulation with gonadotropins. N Engl J Med 2000;343:2-7.

41 Guzick DS, Carson SA, Coutifaris C, Overstreet JW, Factor-Litvak P, Steinkampf MP, et al. Efficacy of superovulation and intrauterine insemination in the treatment of infertility. N Engl J Med 1999;340:177-83.
42 National Institute for Health and Care Excellence. Fertility: assessment and treatment for people with fertility problems. NICE, 2013.

Accepted: 17 November 2014

\section{Cite this as: BMJ 2015;350:g7771}

This is an Open Access article distributed in accordance with the Creative Commons Attribution Non Commercial (CC BY-NC 4.0) license, which permits others to distribute, remix, adapt, build upon this work non-commercially, and license their derivative works on different terms, provided the original work is properly cited and the use is non-commercial. See: http://creativecommons.org/licenses/by-nc/4.0/. 


\section{Tables}

Table 1| Baseline characteristics of couples. Values are numbers (percentages) unless stated otherwise

\begin{tabular}{|c|c|c|c|}
\hline Characteristic & IVF-SET ( $n=201)$ & IVF-MNC ( $n=194)$ & IUI-COH (n=207) \\
\hline Mean (SD) age of female partner, years & $33(3.39)$ & $33(3.50)$ & $34(3.67)$ \\
\hline Primary subfertility & $160(80)$ & $141(73)$ & $157(76)$ \\
\hline Median (IQR) duration of subfertility, years & $2.13(1.73-3.01)$ & $2.14(1.77-2.81)$ & $2.30(1.82-3.13)$ \\
\hline White & $182(91)$ & $163(84)$ & $178(86)$ \\
\hline Smoking & $45(22)$ & $35(18)$ & $46(22)$ \\
\hline Median (IQR) body mass index, $\mathrm{kg} / \mathrm{m}^{2}$ & $23(21-26)$ & $23(21-25)$ & $23(21-26)$ \\
\hline Median (IQR) total motile sperm count $\left(\times 10^{6}\right)$ & $51(25-100)$ & $53(25-126)$ & $59(30-124)$ \\
\hline \multicolumn{4}{|l|}{ Diagnosis of subfertility: } \\
\hline Unexplained & $183(91)$ & $173(89)$ & $189(91)$ \\
\hline Male factor & $18(9)$ & $21(11)$ & $18(9)$ \\
\hline Mean (SD) Hunault score* & $20(6.56)$ & $21(6.83)$ & $19(6.38)$ \\
\hline
\end{tabular}

IQR=interquartile range; IUI-COH=intrauterine insemination with controlled ovarian hyperstimulation; IVF-MNC=in vitro fertilisation in modified natural cycle; IVF-SET=in vitro fertilisation with single embryo transfer.

${ }^{*}$ Calculated according to formula by Van der Steeg et al $2007{ }^{7}$ 
Table 2| Number of couples per intervention

\begin{tabular}{lccc} 
Treatment & IVF-SET $(\mathbf{n}=\mathbf{2 0 1})$ & IVF-MNC $(\mathbf{n}=\mathbf{1 9 4})$ & IUI-COH $(\mathbf{n}=\mathbf{2 0 7})$ \\
\hline 1 cycle & $177^{*}$ & 166 & 194 \\
\hline 2 cycles & $90^{*}$ & 135 & 173 \\
\hline 3 cycles & $34^{*}$ & 113 & 145 \\
\hline 4 cycles & - & 91 & 125 \\
\hline 5 cycles & - & 72 & 100 \\
\hline 6 cycles & - & 55 & 85 \\
\hline Additional treatment within 12 months & 15 & 63 & 70 \\
\hline
\end{tabular}

$\mathrm{IUI}-\mathrm{COH}=$ intrauterine insemination with controlled ovarian hyperstimulation; IVF-MNC=in vitro fertilisation in modified natural cycle; IVF-SET=in vitro fertilisation with single embryo transfer.

*Data in IVF-SET group is number of fresh cycles started; according to protocol, couples received maximum of three fresh cycles of IVF-SET. 
Table 3| Total number of cycles performed within 12 months

\begin{tabular}{lccc} 
No of cycles & IVF-SET & IVF-MNC & IUI-COH \\
\hline Total No of fresh cycles & 301 & 632 & 822 \\
\hline Total No of frozen cycles & 158 & - & - \\
\hline Total additional fresh IVF/ICSI cycles & 5 & 38 & 68 \\
\hline Total additional frozen IVF/ICSI cycles & 0 & 7 & 17 \\
\hline Total additional IUI cycles & 45 & 61 & 31 \\
\hline
\end{tabular}

ICSI= intracytoplasmic sperm injection; IUI- $\mathrm{COH}=$ intrauterine insemination with controlled ovarian hyperstimulation; IVF-MNC=in vitro fertilisation in modified natural cycle; IVF-SET=in vitro fertilisation with single embryo transfer. 


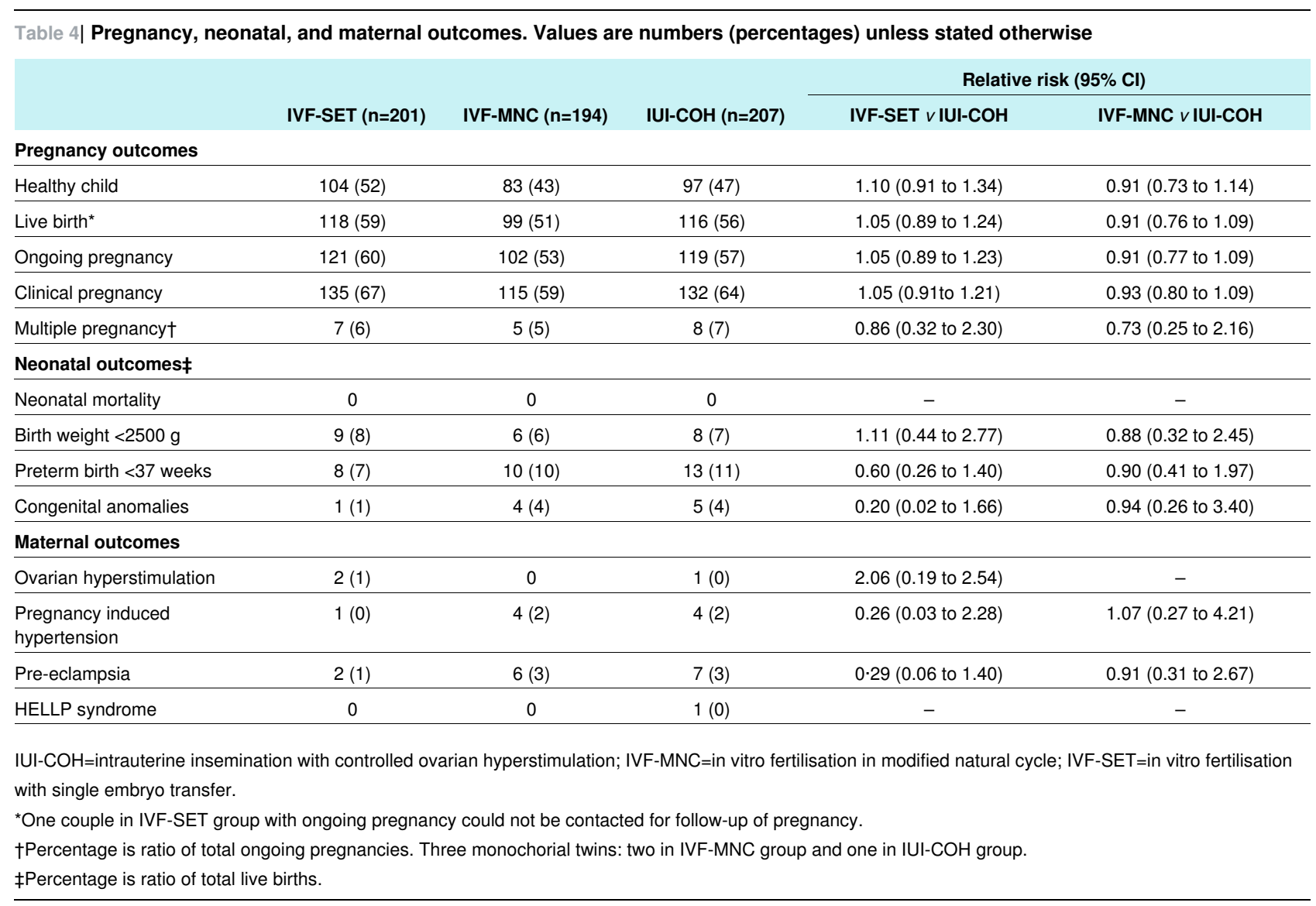




\section{Figures}

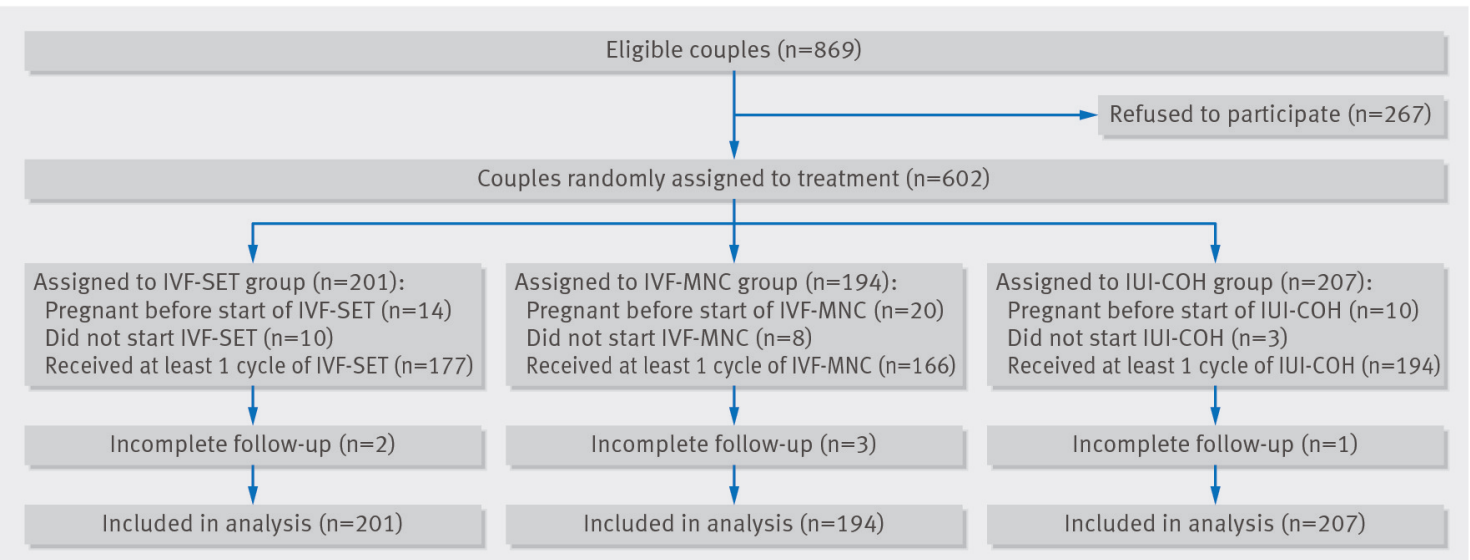

Fig 1 Flow chart of study. IUI-COH=intrauterine insemination with controlled ovarian hyperstimulation; IVF-MNC=in vitro fertilisation in modified natural cycle; IVF-SET=in vitro fertilisation with single embryo transfer

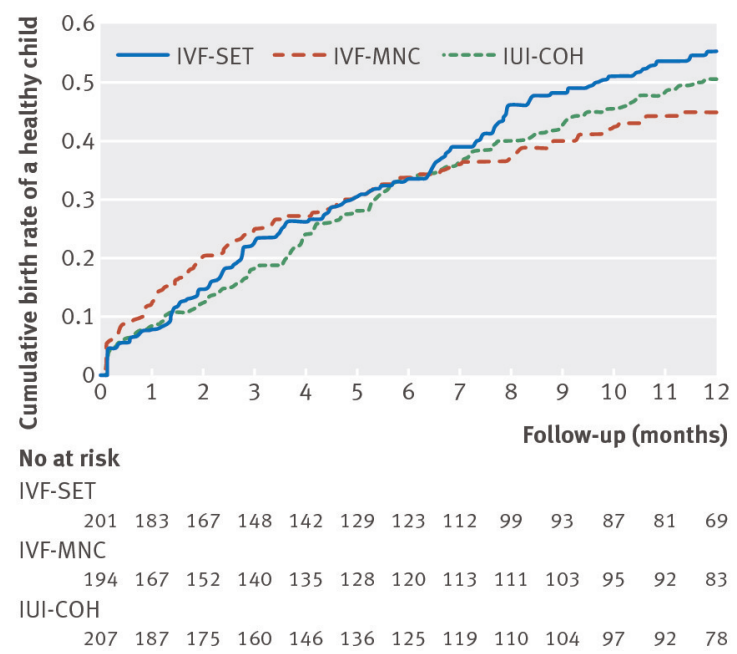

Fig 2 Time to pregnancy resulting in healthy child. IUI-COH=intrauterine insemination with controlled ovarian hyperstimulation; IVF-MNC=in vitro fertilisation in modified natural cycle; IVF-SET=in vitro fertilisation with single embryo transfer 

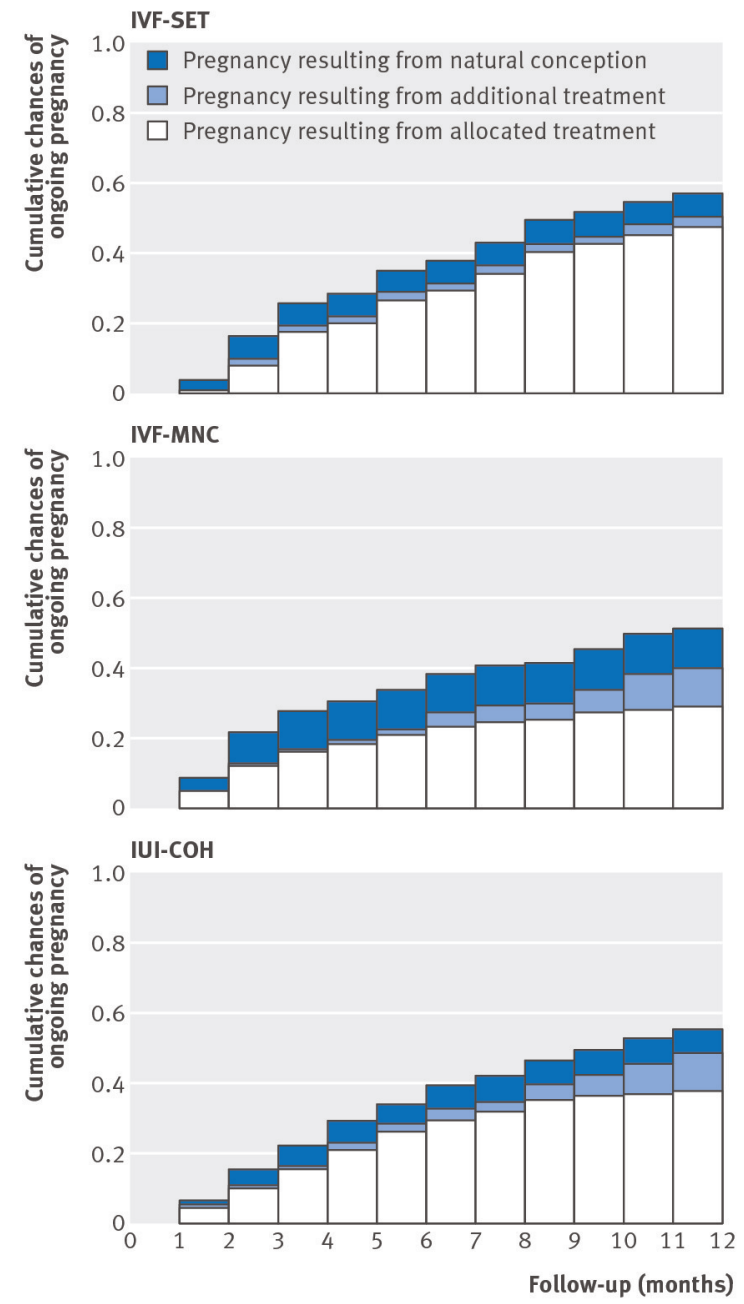

Fig 3 Cumulative chances of ongoing pregnancy. IUI-COH=intrauterine insemination with controlled ovarian hyperstimulation; IVF-MNC=in vitro fertilisation in modified natural cycle; IVF-SET=in vitro fertilisation with single embryo transfer 